\title{
Balancing bistable perception during self-motion
}

\author{
Michiel van Elk • Olaf Blanke
}

Received: 20 June 2012 / Accepted: 24 July 2012

(C) Springer-Verlag 2012

\begin{abstract}
In two experiments we investigated whether bistable visual perception is influenced by passive own body displacements due to vestibular stimulation. For this we passively rotated our participants around the vertical (yaw) axis while observing different rotating bistable stimuli (bodily or non-bodily) with different ambiguous motion directions. Based on previous work on multimodal effects on bistable perception, we hypothesized that vestibular stimulation should alter bistable perception and that the effects should differ for bodily versus non-bodily stimuli. In the first experiment, it was found that the rotation bias (i.e., the difference between the percentage of time that a $\mathrm{CW}$ or $\mathrm{CCW}$ rotation was perceived) was selectively modulated by vestibular stimulation: the perceived duration of the bodily stimuli was longer for the rotation direction congruent with the subject's own body rotation, whereas the opposite was true for the non-bodily stimulus (Necker cube). The results found in the second experiment extend the findings from the first experiment
\end{abstract}

Electronic supplementary material The online version of this article (doi:10.1007/s00221-012-3209-2) contains supplementary material, which is available to authorized users.

M. van Elk $(\bowtie) \cdot$ O. Blanke

Laboratory of Cognitive Neuroscience, Brain Mind Institute,

École Polytechnique Fédérale de Lausanne, Station 19, AI 2101,

1015 Lausanne, Switzerland

e-mail: michiel.vanelk@epfl.ch

O. Blanke

Department of Neurology, University Hospital,

Geneva, Switzerland

O. Blanke

Center for Neuroprosthetics, École Polytechnique Fédérale de

Lausanne, Lausanne, Switzerland and show that these vestibular effects on bistable perception only occur when the axis of rotation of the bodily stimulus matches the axis of passive own body rotation. These findings indicate that the effect of vestibular stimulation on the rotation bias depends on the stimulus that is presented and the rotation axis of the stimulus. Although most studies on vestibular processing have traditionally focused on multisensory signal integration for posture, balance, and heading direction, the present data show that vestibular self-motion influences the perception of bistable bodily stimuli revealing the importance of vestibular mechanisms for visual consciousness.

Keywords Bistable perception - Biological motion . Vestibular processing

\section{Introduction}

When observers are presented with ambiguous visual information, their percept often changes between possible alternative interpretations, a phenomenon that is known as bistable perception. Over the last decades, several studies have shown that multimodal interactions between action and perception (Wohlschlager 2000; Mitsumatsu 2009) and between touch and vision (Blake et al. 2004; Konkle et al. 2009; Butz et al. 2010) lead to systematic changes in bistable perception. For instance, touching a rotating globe affected the perceived rotation direction of a visually presented globe, whose rotation direction was ambiguous (Blake et al. 2004). Similarly, it was found that repeated exposure to tactile motion induces visual motion aftereffects that bias the perceived direction of ambiguous visual motion in the direction of the visual motion aftereffect (Konkle et al. 2009). 
Recently, several stimuli showing a human body or body parts that are characterized by ambiguous visual motion have been described (Troje and McAdam 2010; Hirai et al. 2011). As vestibular stimulation has been shown to modulate perceptual, cognitive, and conscious aspects of bodily processing (Lenggenhager et al. 2008; Lopez et al. 2009, 2010; Ferre et al. 2011), we here investigated whether observer motion, conveyed by vestibular signals, modulates the perception of bistable moving human bodies. Given the functional importance of integrating vestibular with visual motion information for self-motion perception and the prominent neural interactions between vestibular and visual motion processing (e.g., Chowdhury et al. 2009; Liu and Angelaki 2009), one may expect that vestibular information may also influence bistable visual motion perception of human bodies.

In the present study we hypothesized that the seen rotation direction of a bistable rotating body can be modulated by vestibular information regarding passive rotation of one's own body. In two experiments subjects were rotated on a human motion platform while observing bistable stimuli with an ambiguous rotation direction (alternating randomly between clockwise, $\mathrm{CW}$, and counterclockwise, CCW, rotations) and representing bodily (human avatars) or non-bodily control stimuli (Necker cube). If direction-specific vestibular information modulates bistable perception, we should expect that the rotation bias (i.e., the difference between the percentage of time that a $\mathrm{CW}$ or $\mathrm{CCW}$ rotation was perceived) differs between actual $\mathrm{CW}$ and $\mathrm{CCW}$ rotations of the observer.

More specifically, we made the following predictions. First, for bodily stimuli we expected that participants would perceive the rotation direction via a process of embodied perspective taking. Several studies have shown that in spatial perspective taking participants adopt the position and perspective of the observed person (Parsons 1987; Zacks et al. 1999; Arzy et al. 2006; Tadi et al. 2009) and that such own body transformations may occur spontaneously without any imagery instructions (Thirioux et al. 2010). With respect to the perception of bistable stimuli, we accordingly expected that participants would perceive a bistable body more often as rotating in the direction congruent with their own rotation direction. As mental transformation of non-bodily objects has been dissociated from those of bodily objects (i.e., Zacks et al. 2003) and as vestibular stimulation interferes differently with mental transformations of bodily and non-bodily objects (i.e., Lenggenhager et al. 2008), we predicted that bistable perception of a non-bodily stimuli such as the Necker cube would not be influenced or differently influenced by observer rotation (i.e., be perceived more often as rotating in the direction opposite to one's own body).

\section{Methods}

Subjects

In the first experiment 14 subjects participated ( 3 females, mean age $=23.6$ years), and in the second experiment 12 subjects participated ( 5 females, mean age $=21.8$ years). All participants had normal or corrected-to-normal vision. Subjects received $20 \mathrm{CHF}$ for participation. Both experiments were approved by the local ethics committee: La Commission d'ethique de la recherche Clinique de la Faculté de Biologie et de Médecine-at the University of Lausanne, Switzerland. All subjects gave informed consent prior to participation and were fully debriefed after the experiment. The study was conducted in accordance with the declaration of Helsinki.

\section{Stimuli}

In the first experiment 3 different stimuli were used, representing a female avatar, a male avatar, and a Necker cube (see left side of Fig. 1 for example stimuli and supplementary material online). Both female and male avatars were included as they differed strongly in the number of perceptual features (e.g., hair, breasts) that might play a role in bistable perception. For the avatars we used 3D models adopting a standing posture with the arms stretched out that were available from open source libraries and that have been used previously (Troje and McAdam 2010). For the Necker cube, we used a tilted wireframe. In the second experiment we only used the female avatar (as in Experiment 1), but shown in either a vertical or horizontal position and as rotating either around the yaw axis or around the roll axis (see left side of Fig. 2). These 3D models were imported in Blender (http://www.blender.org/), which is an open source 3D rendering software package. The figures were rendered orthographically with the camera viewpoint placed in the middle. The rotation speed of all stimuli was $90 \%$, in line with previous studies on bistable perception (e.g., Blake et al. 2004; Troje and McAdam 2010). The 3D figures used to render the $2 \mathrm{D}$ stimuli were always rotated clockwise, and this resulted in 2D stimuli with a bistable rotation direction. Rendered images were presented at a resolution of $1,024 \times 768$ pixels, and the avatars were about $250 \times 400$ pixels in size, whereas the Necker cube was about $350 \times 350$ pixels in size.

\section{Experimental setup}

The experiment was conducted in complete darkness in a sound-shielded room in which a human motion platform was placed. A chair was mounted on a beam platform 

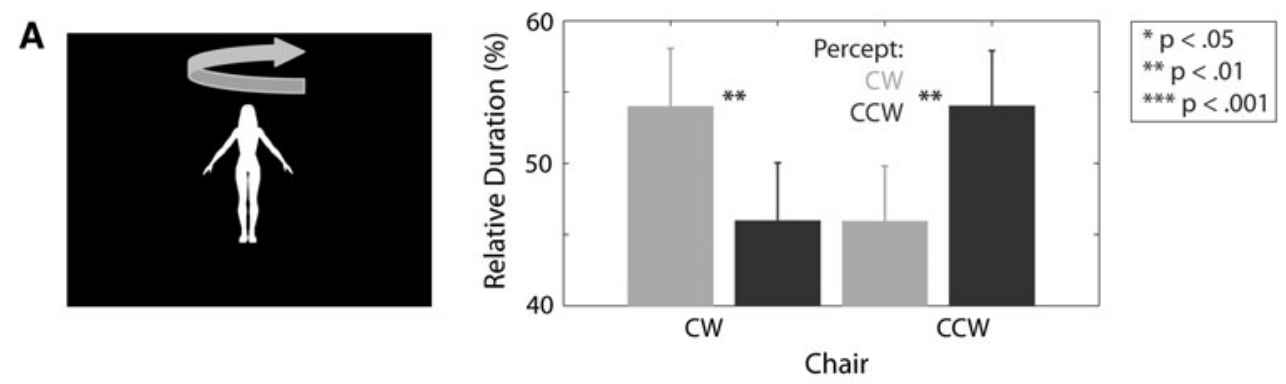

B
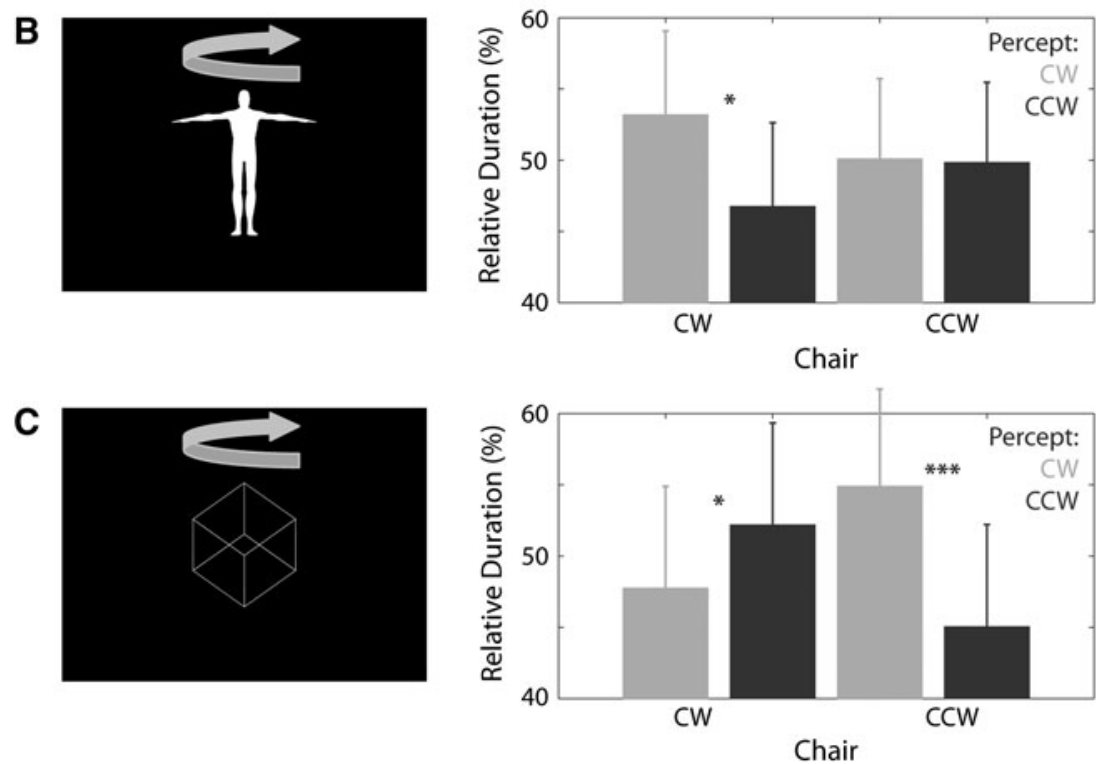

Fig. 1 Example stimuli and effects of rotation on bistable perception in Experiment 1. Left side Example snapshots of the video stimuli used in Experiment 1, representing a female avatar (a), a male avatar (b), and the Necker cube (c). The gray arrow indicates the axis of rotation: yaw rotations for all stimuli. Right side The graphs represent the relative duration that a $\mathrm{CW}$ rotation (light bars) or CCW rotation (dark bars) was perceived, with respect to the rotating direction of the chair (CW: left side of graph, CCW: right side of graph). a Data in

(diameter $=200 \mathrm{~cm}$ ) fixed on an electrical engine. The electrical engine was digitally servo-controlled (PCI-7352) and its software controller allowed for a precise positioning $( \pm 0.01)$ and for the execution of rotation profiles expressed as sequences of positions at $100 \mathrm{~Hz}$. All the rotations were carried out around the yaw axis. A $22^{\prime \prime}$ computer screen was mounted on the chair with an adjustable but fixed arm, placed at $40 \mathrm{~cm}$ away from the subject's eyes. Images were generated by an onboard computer which was controlled from the outside by network desktop sharing (WIFI). A rumble pad PC game controller (Saitek P2600) was connected to the computer to measure subjects' responses.

Subjects were seated in the chair wearing seatbelts, with their head aligned to their body's z-axis and precisely located in the center of rotation. An infrared surveillance camera was mounted on the chair showing the face of the subjects and allowed to monitor participants' eye movements. Another infrared camera displayed the chair itself. response to the female avatar stimulus. b Data from the male avatar stimulus. c Data in response to the Necker cube. Please note that $p$ values indicate whether the observed rotation bias (i.e., the difference between the percentage of time that a $\mathrm{CW}$ or $\mathrm{CCW}$ rotation was perceived) is different from the rotation bias obtained from the permuted distribution (i.e., $p$ values reflect whether the observed rotation bias is significantly affected by the actual rotation profile of the chair)

During the experiment, communication was possible between the subject and the experimenter. The experiment was conducted in complete darkness.

\section{Experimental procedure}

At the beginning of the experiment, subjects were instructed to track the perceived rotation direction of the stimuli on the screen. They were told that the rotation direction of the stimulus varied randomly and instructed to hold the left button pressed if they perceived the stimulus as rotating in a counterclockwise direction and the right button if they perceived the stimulus as rotating in a clockwise direction. At the beginning of each block, subjects practiced while the chair was standing still. After it was established that the subjects understood the task and were able to perceive the stimulus in both directions, the experiment was started. 
During each block, the motion platform generated 20 clockwise and 20 counterclockwise rotations in a random order. Each rotation profile consisted of four phases starting with a 2,000-ms acceleration to a speed of $45 \%$, 2,000-3,000 ms of constant speed, 2,000-ms deceleration to $0 \%$ followed by a 2,000-3,000-ms no-movement interval. The rotation profile and speed were based on previous studies, showing that this acceleration/deceleration profile results in a robust oculogyral illusion (Clark and Stewart 1968; Carriot et al. 2011), and on own pilot studies with the present paradigm showing strongest effects of rotation on bistable perception when the rotation speed changed dynamically. The constant movement and no-movement interval varied pseudorandomly between 2,000 and 3,000 $\mathrm{ms}$ in steps of $100 \mathrm{~ms}$ to prevent the subject from anticipating the onset and offset of the rotations. For each subject a different 6-minute sequence of rotation profiles was generated, and each subject was always rotated using the same rotation profile for each block.

In different blocks, subjects were required to report the perceived rotation direction of the visual stimulus on the screen while they received passive whole-body rotations along the yaw axis. During each block the stimulus was continuously presented on the screen for $6 \mathrm{~min}$, aligned with the onset of the rotating profile of the chair, resulting in a total number of 90 chair rotations. During the
Fig. 2 Example stimuli and effects of rotation on bistable perception in Experiment 2. Left side: Example snapshots of the video stimuli used in Experiment 2, representing a female avatar in a vertical orientation $(\mathbf{a}, \mathbf{c})$ or a horizontal orientation $(\mathbf{b}, \mathbf{d})$. The gray arrow indicates the axis of rotation: yaw rotations $(\mathbf{a}, \mathbf{d})$ or roll rotations $(\mathbf{b}, \mathbf{c})$. Right side Graphs represent the relative duration that a $\mathrm{CW}$ rotation (light bars) or CCW rotation (dark bars) was perceived, with respect to the rotating direction of the chair (CW: left side of graph, CCW: right side of graph). a Data in response to the female avatar in a vertical position and rotating along the yaw axis (similar to the first stimulus in Experiment 1).

b Data in response to the female avatar in a horizontal position and rotating along the roll axis. c Data in response to the female avatar in a vertical position and rotating along the roll axis.

d Data in response to the female avatar in a horizontal position and rotating along the yaw axis
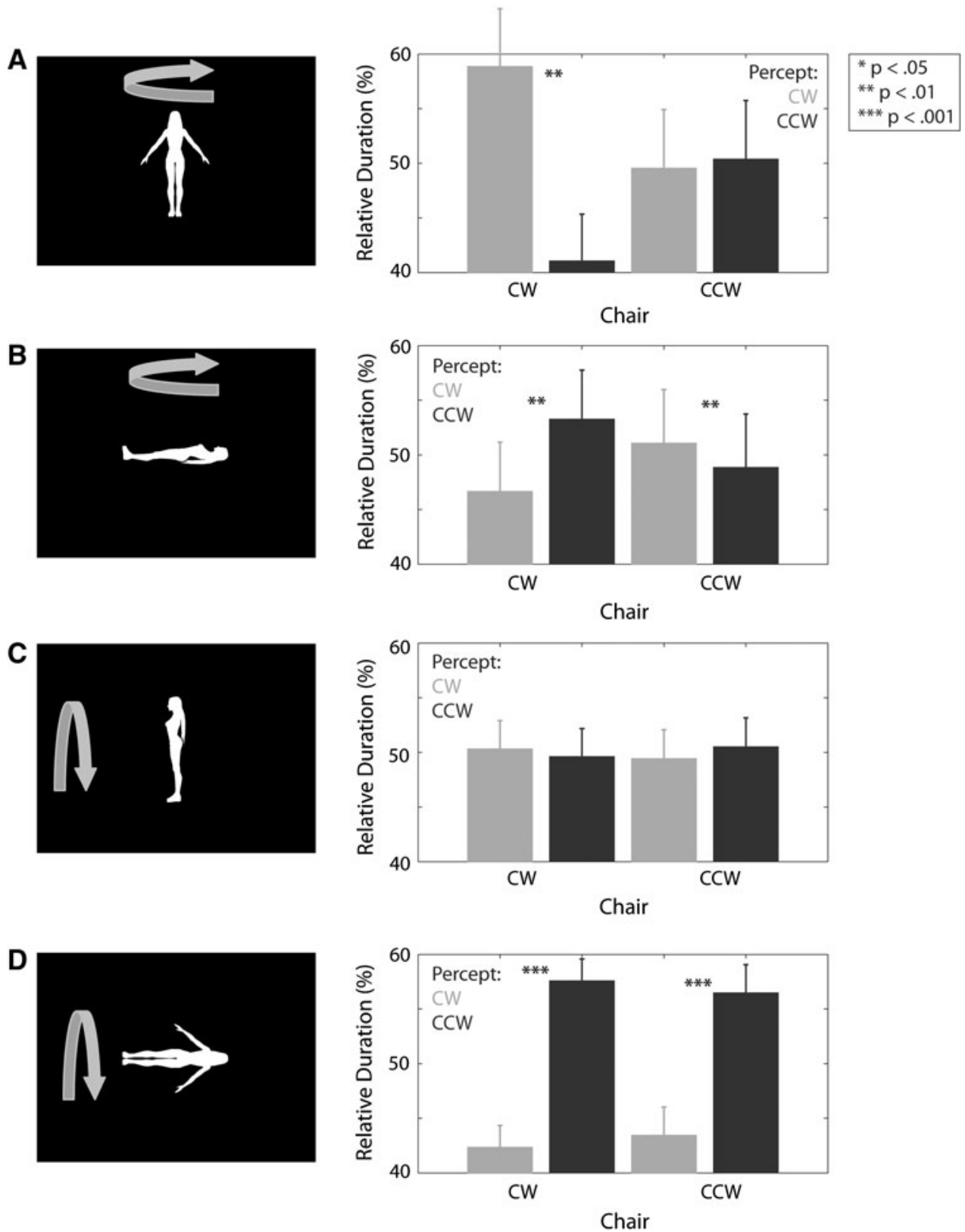
experiment white noise was presented over the subjects' headphones and a black blanket covered the chair, to avoid the participant from inferring the rotation direction based on auditory or visual cues (residual light emanating from the stimulus display). Stimulus presentation and button press responses were controlled and stored for offline analysis using Presentation software version 12.2 (Neurobehavioral Systems, Davis, CA, USA).

\section{Analysis}

Based on previous work on bistable stimulus perception, we focused our analysis on the total number of switches and the relative duration of perceived $\mathrm{CW}$ and $\mathrm{CCW}$ rotations of the stimulus (Wohlschlager 2000; Blake et al. 2004; Jackson et al. 2008; Butz et al. 2010). To assess whether the rotation direction of the participant's body had an effect on the perceived rotation direction of the different bistable stimuli, we calculated the percentage of time that subjects perceived the stimulus as rotating in the $\mathrm{CW}$ or $\mathrm{CCW}$ direction for both rotation directions of the chair (CW vs. CCW). Thus, for all $\mathrm{CW}$ and $\mathrm{CCW}$ chair rotations, we obtained the percentage of time that subjects perceived the stimulus as rotating in a $\mathrm{CW}$ direction and a $\mathrm{CCW}$ direction. The difference between the percentage of time that a CW or a CCW direction of the visual stimuli was perceived has been used previously under different experimental conditions (Blake et al. 2004; Jackson et al. 2008) and provides a measure of the "rotation bias": (1) a positive rotation bias reflects a preference for perceiving the visual stimulus as rotating in a $\mathrm{CW}$ direction, (2) a rotation bias of zero reflects no preference for perceiving the visual stimulus as rotating in a $\mathrm{CW}$ or $\mathrm{CCW}$ direction, and (3) a negative rotation bias reflects a preference for perceiving the visual stimulus as rotating in a $\mathrm{CCW}$ direction. If the rotation direction of the chair has an effect on the perceived rotation direction of the stimulus, we should expect the rotation bias to differ between $\mathrm{CW}$ and $\mathrm{CCW}$ chair rotations.

For each subject, we calculated the rotation bias separately for the 2 different yaw rotation directions of the chair (CW vs. $\mathrm{CCW}$ ), and for each visual stimulus (3 in Experiment 1 and 4 in Experiment 2). To statistically evaluate the observed effects, we used two methods. First, a random permutation test was conducted on the rotation biases. To this end, a randomization distribution was created by randomly permuting the rotation profiles across different subjects 1,000 times. The observed rotation biases were compared to the 95th percentile of the permuted distribution, and the $p$ value was calculated as the proportion of values of the permuted distribution that was equal to or bigger than the observed rotation bias. Note that if the observed rotation biases are significantly induced by the rotation direction of the chair, we should expect the observed rotation biases to lie outside the 95 th percentile of the permuted distribution and a resulting $p$ value of $p<.05$. To correct for multiple comparisons, a Bonferroni correction was applied. Second, a classical analysis of variance was conducted by analyzing the percentage of time that subjects perceived the stimulus as rotating in a $\mathrm{CW}$ or $\mathrm{CCW}$ direction for both rotation directions of the chair ( $\mathrm{CW}$ vs. $\mathrm{CCW}$ ). In the first experiment, a 3 (Stimulus Type: Female Avatar, Male Avatar, Necker Cube) $\times 2$ (Chair Direction: $\mathrm{CW}$ vs. $\mathrm{CCW}) \times 2$ (Perceived Direction: $\mathrm{CW}$ vs. $\mathrm{CCW})$ repeated measures ANOVA was used. In the second experiment, a 4 (Stimulus Type: Horizontal-Yaw, Horizontal-Roll, Vertical-Yaw, Vertical-Roll) $\times 2$ (Chair Direction: $\mathrm{CW}$ vs. $\mathrm{CCW}) \times 2$ (Perceived Direction: $\mathrm{CW}$ vs. $\mathrm{CCW}$ ) repeated measures ANOVA was used. The rationale for including the ANOVA is that it allows for a more direct assessment of the central hypothesis, that is, effects of observer rotation on bistable perception should be reflected in an interaction between Chair Direction and Perceived Direction. In addition, if the rotation bias differs between the different stimuli, an interaction is expected between Stimulus Type, Chair Direction, and Perceived Direction.

\section{Results and discussion}

\section{Experiment 1}

On average subjects perceived $47.6(\mathrm{SD}=40.3)$ changes in the rotation direction of the female avatar, 25.8 $(\mathrm{SD}=34.8)$ changes in the rotation direction of the male avatar, and $12.8(\mathrm{SD}=3.9)$ changes in the rotation direction of the Necker cube.

For all bistable stimuli, the rotation bias (i.e., the difference between the percentage of time that a $\mathrm{CW}$ or $\mathrm{CCW}$ rotation was perceived) was modulated by the rotation direction of the chair (see Fig. 1). Subjects perceived both bodily stimuli (female and male silhouette) relatively longer as rotating in the direction that was congruent with the passive rotation direction of their body. In contrast, subjects perceived the Necker cube relatively longer as rotating in the direction that was opposite to the passive rotation direction of their own body.

Statistical analysis using the permutation approach confirmed that the observed rotation bias differed from the randomization distribution for three of the six tested conditions: for the female avatar for $\mathrm{CW}$ rotations $(p<.01)$ and CCW rotations $(p<.01)$ and for the Necker cube for $\mathrm{CCW}$ rotations $(p<.001)$. Although for the male avatar a trend was observed for $\mathrm{CW}$ rotations $(p<.05)$ and for the Necker cube for $\mathrm{CW}$ rotations $(p<.05)$, these effects did not survive correction for multiple comparisons. 
Statistical analysis using a repeated measures ANOVA revealed a significant interaction between Stimulus Type, Chair Direction, and Perceived Direction, $F(2,26)=5.7$, $p<.01, \eta^{2}=.30$. This interaction provides direct support for the observation that the effects of vestibular stimulation on bistable perception differ between the different stimuli. Post hoc tests using a 2 (Chair Direction: $\mathrm{CW}$ vs. $\mathrm{CCW}) \times 2$ (Perceived Direction: CW vs. CCW) revealed a marginally significant interaction between Chair Direction and Perceived Direction for the female avatar, $F(1,13)=4.0$, $p=.06, \eta^{2}=.24$, reflecting that the perceived rotation direction of the bistable bodily stimulus was modulated by the rotation direction of the chair. For the Necker cube, a significant interaction was observed between Chair Direction and Perceived Direction, $F(1,13)=8.6, p=.01, \eta^{2}=.40$, reflecting that the Necker cube was perceived more often as rotating in the direction opposite to the rotation direction of the chair. For the male avatar, no significant effects were observed in the post hoc test $(F<1)$.

The results of the first experiment show that rotatory vestibular cues alter bistable perception and that this effect differs for bodily and non-bodily bistable cues. For the two bodily stimuli that were shown on the screen, the perceived duration tended to be longer when the perceived direction of stimulus rotation matched the participant's yaw rotation direction (this was only significant for the female avatar). This was different for a non-bodily stimulus often used in bistable perception studies, the Necker cube, which was perceived as rotating relatively longer in the rotation direction that was opposite to the subjects' own body rotation direction. This shows that vestibular signals from the semicircular canals influence the perception of visual bistable stimuli, that this effect is direction-selective, and that it depends on the type of visual object shown.

\section{Experiment 2}

In a second experiment we investigated whether the vestibular effect on bistable perception depends on (1) the congruency of the orientation of the depicted visual stimulus on the screen (horizontal vs. vertical) with the participant's orientation and/or on (2) the congruency of the depicted visual rotation axis of the avatar (roll rotation vs. yaw rotation) with respect to the participant's actual rotation axis. We thus carried out a $2 \times 2$ factorial design with the factors orientation and rotation while our participants were always exposed to yaw rotations and observed a bistable female avatar (see left side of Fig. 2). ${ }^{1}$ Based on

\footnotetext{
${ }^{1}$ In the second experiment only the female avatar was used, as this stimulus showed the strongest effects in the first experiment, likely because the stimulus contained more depth-cues (i.e. breasts and hair) to disambiguate the rotation direction.
}

pilot experiments and the data of Experiment 1, we expected a maximal effect on bistable perception if the orientation and rotation axis of the observed avatar are congruent with the observer (i.e., relatively longer perceived duration in the direction that is congruent with one's own rotation direction). In contrast, in the case the avatar is depicted in a horizontal position and rotating around its roll axis, we speculated that bistable perception is not altered or altered in the opposite direction (as now neither the depicted avatar's rotation direction nor orientation is congruent with the participant's rotation direction and orientation). For the two cases, where either rotation direction or orientation was congruent, we expected intermediate effects on bistable perception.

In the second experiment, 12 new subjects perceived on average $33(\mathrm{SD}=45.0)$ changes in the rotation direction when the female avatar was presented in a vertical orientation and rotated around the yaw axis (as in Experiment 1), 54.0 $(\mathrm{SD}=47.2)$ changes in the rotation direction when the female avatar was presented in a horizontal orientation and rotated around the roll axis, $91.4(\mathrm{SD}=43.5)$ changes when the avatar was presented in a vertical orientation and rotated around the roll axis, and 80.1 $(\mathrm{SD}=42.7)$ changes when the avatar was presented in a horizontal position and rotated around the yaw axis.

Similar to the first experiment, subjects perceived the vertical avatar spinning around the yaw axis relatively longer as rotating in the direction that was congruent with their own rotation direction (Fig. 2a). In contrast, subjects perceived the horizontal avatar rotating around the roll axis relatively longer as rotating in the direction that was opposite to their own rotation direction (Fig. 2b), resembling the pattern observed for the Necker cube in Experiment 1 . The rotation bias was not modulated by the rotation direction of the chair when the vertical avatar rotated on the roll axis or when the horizontal avatar rotated on the yaw axis (Fig. 2c, d). Thus, participants' yaw rotation has an effect on bistable perception only if the visual stimulus seemed to rotate around the visual yaw axis (i.e., when subjects observed an avatar in a vertical orientation rotating around its "yaw" axis or when they observed an avatar in a horizontal orientation rotating around its "roll" axis).

Statistical analysis using the permutation approach confirmed that the observed rotation bias differed from the randomization distribution for the avatar in a vertical position rotating around the yaw axis (Fig. 2a) for CW rotations $(p<.01)$ and for the female avatar in a horizontal position rotating around the roll axis (Fig. $2 \mathrm{~b}$ ) for $\mathrm{CW}$ $(p<.01)$ and $\mathrm{CCW}$ rotations $(p<.01)$.

Statistical analysis using a repeated measures ANOVA revealed a significant interaction between Stimulus Type, Chair Direction, and Perceived Direction, $F(3,33)=4.1$, $p<.05, \eta^{2}=.26$. This interaction provides further support 
for the finding that the effects of vestibular stimulation on bistable perception differ between the different stimuli. Post hoc tests using a separate 2 (Chair Direction: CW vs. $\mathrm{CCW}) \times 2$ (Perceived Direction: $\mathrm{CW}$ vs. $\mathrm{CCW}$ ) repeated measures ANOVA for each of the 4 different stimuli revealed a significant interaction between Chair Direction and Perceived Direction for the female avatar in a horizontal position rotating around the roll axis, $F(1,11)=$ $6.2, p<.05$. For the other stimuli, the post hoc ANOVAs did not yield significant results.

\section{Discussion}

The present study shows that vestibular information has an effect on bistable perception that is dependent on (1) the rotation direction of the observer's body, (2) whether a bodily or a non-bodily bistable stimulus is presented, and (3) the congruency of the orientation and the rotation axis of the rotating stimulus with the motion profile of the observer. The effect of vestibular stimulation on bistable perception extends previous studies that have shown effects of action and touch on bistable perception (Wohlschlager 2000; Blake et al. 2004; Konkle et al. 2009; Mitsumatsu 2009; Butz et al. 2010), to the domain of visuo-vestibular interaction. Whereas in a previous study it was found that touching a rotating globe affected the perceived rotation direction of a visually presented globe, whose rotation direction was ambiguous (Blake et al. 2004), the present study shows that rotation of the entire observer affects the perceived rotation of human bodies and objects.

Upright human bodies rotating along the same axis as the observer tended to be perceived more often as rotating in the same direction as the observer's body. That is, CW observer rotation biased the perceived body rotation in the $\mathrm{CW}$ direction and vice versa for $\mathrm{CCW}$ rotations. We argue that this effect reflects an automatic process of embodied perspective taking, in which subjects determine the rotation direction by adopting the position and perspective of the avatar. Related mechanisms have been studied using mental own body transformations, in which subjects are explicitly asked to imagine themselves at the position of a human figure that is presented in many different visual orientations and views (Parsons 1987; Zacks et al. 1999; Arzy et al. 2006; Tadi et al. 2009) and in studies showing that visual motion signals and action observation influence the body and movement representation systems in the brain (Calvo-Merino et al. 2005; Chiavarino et al. 2007; van Elk et al. 2008; Serino et al. 2010). Based on these findings, it has been argued that observed movements are mapped directly on the motor repertoire of the observer via a process of "intermodal matching" or "motor resonance" (Meltzoff and Moore 1997; Wohlschläger et al. 2003;
Schütz-Bosbach and Prinz 2007). A similar mechanism may be involved in the present study, in which observed and felt body rotations recruit shared motion representations, thereby extending the intermodal matching account to the visuo-vestibular domain.

This "embodiment" explanation is further supported by the findings of the second experiment, showing that the effect of own body rotation on bistable perception depended on (1) the congruence between the observer's body orientation on the motion platform and the orientation of the observed body on the screen and (2) the congruence between the axis of rotation of the observer's body and the observed body's axis of rotation. Only when both the orientation and rotation direction of the observed avatar were congruent with the observer's, the observed body tended to be perceived as rotating relatively longer in the direction of the observer's body (i.e., one's own body). Incongruent orientation or rotation axis resulted in a different or in no rotation bias. These findings further qualify the interaction between observer motion and bistable perception and are consistent with previous studies that have shown a visual processing advantage for bodies aligned with the observer's body axis orientation (Reed et al. 2003; Troje and Westhoff 2006), effects of the observer's body orientation on the visual judgments of human bodies (Lobmaier and Mast 2007; Lopez et al. 2009), and facilitated biological motion perception when the displayed movements or bodies are familiar (for review, see: Klopfer 1991; SchützBosbach and Prinz 2007; Sebanz and Shiffrar 2009). Thus, the present data suggest that vestibular information influences the bistable perception of a human avatar in a direction-specific fashion only if the observed body is in the same orientation and rotates around the same axis, via a process of facilitated perspective taking or embodiment.

For stimuli that did not afford a process of embodied perspective taking, opposite effects of vestibular stimulation on bistable perception were observed. One of the most classical stimuli used to study bistable visual perception, the Necker cube, was perceived relatively longer as rotating in the direction opposite to one's own body rotation. That is, CCW observer rotation biased the perceived Necker cube rotation in the $\mathrm{CW}$ direction and vice versa. The size of the rotation bias was comparable to those observed for tactile effects on visual motion judgments (e.g., Butz et al. 2010), but differed with respect to its direction. Thus, a congruent directional rotation bias was observed for tactile and visual drum rotations on the forearm (Blake et al. 2004), whereas we observed an opposite directional rotation bias for observer rotation paired with a bistable Necker cube rotation. A similar effect was observed when a human silhouette was presented in a horizontal position and rotating in the roll axis, in which case subjects perceived the bistable body 
relatively longer as rotating in a direction opposite to their own body. We speculate that these findings are compatible with directional effects observed during visuo-vestibular illusions such as the oculogyral illusion, in which a stationary object is perceived as moving in space counter to the preceding observer rotation (Graybiel and Hupp 1946; Mergner et al. 1992). We argue that if embodied perspective taking is not possible (as is the case for incongruent avatars and non-bodily objects such as the Necker cube), participants are biased to perceive an object as rotating in a direction opposite to their own body movement.

The finding that the direction of the effect of vestibular stimulation on bistable perception depends on the type of stimulus that is presented suggests a possible hierarchical processing of bistable stimulus information. Given the inherent difficulty in determining the relative rotation direction of an ambiguous stimulus, subjects may first attempt to use an embodied perspective taking strategy. If this approach is not afforded by the stimuli, participants revert to a strategy whereby they determine the rotation direction as if viewed from an external perspective. In addition, we would like to point out that perceptual complexity differences between stimuli cannot account for the differences observed between bodily and non-bodily stimuli. That is, in the second experiment similar effects as those for the Necker cube were observed when a bodily stimulus was tilted in the horizontal plane and rotating along the horizontal axis. Thus, the crucial factor in modulating the effects of vestibular stimulation on bistable perception is the similarity between the observed and felt bodily rotations, rather than the visual complexity of the stimulus involved.

The present findings on bistable perception of rotating stimuli can also be compared with previous studies, showing that vestibular stimulation, as induced by caloric vestibular stimulation, can influence binocular rivalry (Miller et al. 2000; Ngo et al. 2007, 2008). It was found for instance that left hemispheric caloric vestibular stimulation changes the predominance of subjects' perceptions in binocular rivalry using horizontal and vertical gratings and the Necker cube (Miller et al. 2000). However, the observed effects were not specific to caloric vestibular stimulation and differed between subjects (Miller et al. 2000). The present study differs in important respects from these previous findings. That is, we observed a directionspecific effect of vestibular stimulation that depended on the type of object that was presented (i.e., bodily vs. nonbodily stimulus). Whereas caloric vestibular stimulation results in an unnatural activation of the vestibular organ (as well as other undesired side effects) and the activation of a large network of cortical structures, involving both visual and multisensory association areas (Lopez and Blanke 2011), passive whole-body rotation provides a natural way to selectively stimulate the semicircular canals.
We speculate that the selective modulation of bistable perception by passive own body rotations reflects a topdown influence of vestibular signals on higher visual areas involved in visual motion processing, such as area MT and V5 (Vaina et al. 2001; Born and Bradley 2005; Tailby et al. 2010; Tadin et al. 2011). This suggestion is supported by previous studies showing a top-down influence of multisensory information on bistable perception in area MT (Vaina et al. 2001; Blake et al. 2004; Born and Bradley 2005) and by studies underlining the neural interactions between vestibular and visual motion processing (e.g., Chowdhury et al. 2009; Liu and Angelaki 2009).

In sum, this study shows that vestibular semicircular canal signals influence the perception of visual bistable stimuli, that the effect is direction-selective, and that it depends on the type of stimulus and on the orientation and rotation axis of the visual stimulus. Studies in the domain of vestibular processing have mainly focused on the integration of multisensory signals for body posture, balance, and heading direction (Green and Angelaki 2010). Our data reveal that passive own body displacements due to vestibular stimulation systematically modulate bistable perception, which is in line with the functional importance of integrating vestibular with visual motion information (Young et al. 1973; Buttner and Henn 1981). A more recent line of work has started investigating the effects of vestibular signals on mental imagery (Lenggenhager et al. 2008) and other aspects of cognition (Berthoz et al. 1995) as well as bodily self-consciousness (Lopez et al. 2009, 2010; Ferre et al. 2011; Ionta et al. 2011). The present study extends the latter line of research showing that lowlevel vestibular signals regarding self-motion influence mechanisms of visual consciousness that are further modulated by the type of bistable object that is seen.

Acknowledgments We thank Bruno Herbelin for assistance in creating the 3D stimuli and Mario Prsa for assistance in setting up the experiment on the motion platform. The present study was supported by the Marie Curie Intra European Fellowship within the Seventh European Community Framework Program (IEF grant 252713 to MVE). OB is supported by the Swiss National Science foundation, the European Science Foundation, and the Fondation Bertarelli.

\section{References}

Arzy S, Thut G, Mohr C, Michel CM, Blanke O (2006) Neural basis of embodiment: distinct contributions of temporoparietal junction and extrastriate body area. J Neurosci 26:8074-8081. doi: 10.1523/JNEUROSCI.0745-06.2006

Berthoz A, Israel I, Georges-Francois P, Grasso R, Tsuzuku T (1995) Spatial memory of body linear displacement: what is being stored? Science 269:95-98

Blake R, Sobel KV, James TW (2004) Neural synergy between kinetic vision and touch. Psychol Sci 15:397-402. doi:10.1111/ j.0956-7976.2004.00691.x 
Born RT, Bradley DC (2005) Structure and function of visual area MT. Annu Rev Neurosci 28:157-189. doi:10.1146/annurev.neuro.26. 041002.131052

Buttner U, Henn V (1981) Circularvection-psychophysics and single-unit recordings in the monkey. Ann NY Acad Sci 374: 274-283

Butz MV, Thomaschke R, Linhardt MJ, Herbort O (2010) Remapping motion across modalities: tactile rotations influence visual motion judgments. Exp Brain Res 207:1-11. doi:10.1007/s00221$010-2420-2$

Calvo-Merino B, Glaser DE, Grezes J, Passingham RE, Haggard P (2005) Action observation and acquired motor skills: an FMRI study with expert dancers. Cereb Cortex 15:1243-1249. doi:10. 1093/cercor/bhi007

Carriot J, Bryan A, DiZio P, Lackner JR (2011) The oculogyral illusion: retinal and oculomotor factors. Exp Brain Res 209:415423. doi:10.1007/s00221-011-2567-5

Chiavarino C, Apperly IA, Humphreys GW (2007) Exploring the functional and anatomical bases of mirror-image and anatomical imitation: the role of the frontal lobes. Neuropsychologia 45:784-795. doi:10.1016/j.neuropsychologia.2006.08.007

Chowdhury SA, Takahashi K, DeAngelis GC, Angelaki DE (2009) Does the middle temporal area carry vestibular signals related to selfmotion? J Neurosci 29:12020-12030. doi:10.1523/JNEUROSCI. 0004-09.2009

Clark B, Stewart I (1968) Comparison of sensitivity for the perception of bodily rotation and the oculogyral illusion. Percept Psychophys 3:253-256

Ferre ER, Bottini G, Haggard P (2011) Vestibular modulation of somatosensory perception. Eur J Neurosci 34:1337-1344. doi: 10.1111/j.1460-9568.2011.07859.x

Graybiel A, Hupp D (1946) The oculo-gyral illusion: a form of apparent motion which may be observed following stimulation of the semicircular canals. J Avian Med Surg 17:3-27

Green AM, Angelaki DE (2010) Multisensory integration: resolving sensory ambiguities to build novel representations. Curr Opin Neurobiol 20:353-360. doi:10.1016/j.conb.2010.04.009

Hirai M, Chang DH, Saunders DR, Troje NF (2011) Body configuration modulates the usage of local cues to direction in biological-motion perception. Psychol Sci 22:1543-1549. doi: 10.1177/0956797611417257

Ionta S, Heydrich L, Lenggenhager B et al (2011) Multisensory mechanisms in temporo-parietal cortex support self-location and first-person perspective. Neuron 70:363-374. doi:10.1016/j.neuron. 2011.03.009

Jackson S, Cummins F, Brady N (2008) Rapid perceptual switching of a reversible biological figure. PLoS ONE 3:e3982. doi: 10.1371/journal.pone.0003982

Klopfer DS (1991) Apparent reversals of a rotating mask: a new demonstration of cognition in perception. Percept Psychophys 49:522-530

Konkle T, Wang Q, Hayward V, Moore CI (2009) Motion aftereffects transfer between touch and vision. Curr Biol 19:745-750. doi: 10.1016/j.cub.2009.03.035

Lenggenhager B, Tadi T, Metzinger T, Blanke O (2007) Video ergo sum: manipulating bodily self-consciousness. Science 317:10961099. doi:10.1126/science. 1143439

Lenggenhager B, Lopez C, Blanke O (2008) Influence of galvanic vestibular stimulation on egocentric and object-based mental transformations. Exp Brain Res 184:211-221. doi:10.1007/s00221007-1095-9

Liu S, Angelaki DE (2009) Vestibular signals in macaque extrastriate visual cortex are functionally appropriate for heading perception. J Neurosci 29:8936-8945. doi:10.1523/JNEUROSCI.1607-09.2009

Lobmaier JS, Mast FW (2007) The Thatcher illusion: rotating the viewer instead of the picture. Perception 36:537-546
Lopez C, Blanke O (2011) The thalamocortical vestibular system in animals and humans. Brain Res Rev 67:119-146. doi:10.1016/ j.brainresrev.2010.12.002

Lopez C, Bachofner C, Mercier M, Blanke O (2009) Gravity and observer's body orientation influence the visual perception of human body postures. J Vision 9(1):1-14. doi:10.1167/9.5.1

Lopez C, Lenggenhager B, Blanke O (2010) How vestibular stimulation interacts with illusory hand ownership. Conscious Cogn 19:33-47. doi:10.1016/j.concog.2009.12.003

Meltzoff AN, Moore K (1997) Explaining facial imitation: a theoretical model. Early Dev Parenting 6:179-192

Mergner T, Rottler G, Kimmig H, Becker W (1992) Role of vestibular and neck inputs for the perception of object motion in space. Exp Brain Res 89:655-668

Miller SM, Liu GB, Ngo TT, Hooper G, Riek S, Carson RG, Pettigrew JD (2000) Interhemispheric switching mediates perceptual rivalry. Curr Biol 10:383-392

Mitsumatsu H (2009) Voluntary action affects perception of bistable motion display. Perception 38:1522-1535

Ngo TT, Liu GB, Tilley AJ, Pettigrew JD, Miller SM (2007) Caloric vestibular stimulation reveals discrete neural mechanisms for coherence rivalry and eye rivalry: a meta-rivalry model. Vision Res 47:2685-2699. doi:10.1016/j.visres.2007.03.024

Ngo TT, Liu GB, Tilley AJ, Pettigrew JD, Miller SM (2008) The changing face of perceptual rivalry. Brain Res Bull 75:610-618. doi:10.1016/j.brainresbull.2007.10.006

Parsons LM (1987) Imagined spatial transformation of one's body. J Exp Psychol Gen 116:172-191

Reed CL, Stone VE, Bozova S, Tanaka J (2003) The body-inversion effect. Psychol Sci 14:302-308

Schütz-Bosbach S, Prinz W (2007) Perceptual resonance: actioninduced modulation of perception. Trends Cogn Sci 11:349-355. doi:10.1016/j.tics.2007.06.005

Sebanz N, Shiffrar M (2009) Detecting deception in a bluffing body: the role of expertise. Psychonom Bull Rev 16:170-175. doi: 10.3758/PBR.16.1.170

Serino A, De Filippo L, Casavecchia C, Coccia M, Shiffrar M, Ladavas E (2010) Lesions to the motor system affect action perception. J Cogn Neurosci 22:413-426. doi:10.1162/jocn.2009. 21206

Tadi T, Overney LS, Blanke O (2009) Three sequential brain activations encode mental transformations of upright and inverted human bodies: a high resolution evoked potential study. Neuroscience 159:1316-1325. doi:10.1016/j.neuroscience.2009. 02.012

Tadin D, Silvanto J, Pascual-Leone A, Battelli L (2011) Improved motion perception and impaired spatial suppression following disruption of cortical area MT/V5. J Neurosci 31:1279-1283. doi:10.1523/JNEUROSCI.4121-10.2011

Tailby C, Majaj NJ, Movshon JA (2010) Binocular integration of pattern motion signals by MT neurons and by human observers. J Neurosci 30:7344-7349. doi:10.1523/JNEUROSCI.4552-09. 2010

Thirioux B, Mercier MR, Jorland G, Berthoz A, Blanke O (2010) Mental imagery of self-location during spontaneous and active self-other interactions: an electrical neuroimaging study. J Neurosci 30:7202-7214. doi:10.1523/JNEUROSCI.3403-09.2010

Troje NF, McAdam M (2010) The viewing-from-above bias and the silhouette illusion. i-Perception 1:143-148. doi:10.1068/i0408

Troje NF, Westhoff C (2006) The inversion effect in biological motion perception: evidence for a "life detector"? Curr Biol 16:821-824. doi:10.1016/j.cub.2006.03.022

Vaina LM, Solomon J, Chowdhury S, Sinha P, Belliveau JW (2001) Functional neuroanatomy of biological motion perception in humans. P Natl Acad Sci USA 98:11656-11661. doi:10.1073/pnas. 191374198 
van Elk M, van Schie HT, Hunnius S, Vesper C, Bekkering H (2008) You'll never crawl alone: neurophysiological evidence for experience-dependent motor resonance in infancy. Neuroimage 43:808-814. doi:10.1016/j.neuroimage.2008.07.057

Wohlschlager A (2000) Visual motion priming by invisible actions. Vision Res 40:925-930

Wohlschläger A, Gattis M, Bekkering H (2003) Action generation and action perception in imitation: an instance of the ideomotor principle. Philos T R Soc Lond 358:501-515
Young LR, Dichgans J, Murphy R, Brandt T (1973) Interaction of optokinetic and vestibular stimuli in motion perception. Acta Oto-Laryngol 76:24-31

Zacks J, Rypma B, Gabrieli JD, Tversky B, Glover GH (1999) Imagined transformations of bodies: an fMRI investigation. Neuropsychologia 37:1029-1040

Zacks J, Vettel JM, Michelon P (2003) Imagined viewer and object rotations dissociated with event-related FMRI. J Cogn Neurosci 15:1002-1018. doi:10.1162/089892903770007399 